Pacific Journal of Mathematics

APPROPRIATE CROSS-SECTIONALLY SIMPLE FOUR-CELLS 


\title{
APPROPRIATE CROSS-SECTIONALLY SIMPLE FOUR-CELLS ARE FLAT
}

\author{
Steve Pax
}

\begin{abstract}
When $X$ is a set in $E^{n}$, we let $X_{t}=X \cap H_{t}$-where $H_{t}$ is the horizontal hyperplane in $E^{n}$ of height $t$. In this note, we prove that a 4-cell $B$ in $E^{4}$, such that each nonempty slice $B_{t}$ is either a point or a 3-cell, is flat whenever, for all $t, B_{t}$ is flat in $H_{t}$ and $\mathrm{Bd} B_{t}$ is flat in $\mathrm{Bd} B$.
\end{abstract}

1. Introduction and summary. Throughout, we let $H_{t}$ denote the horizontal hyperplane in $E^{n}$ at height $t$, and when $X$ is a set in $E^{n}$, we let $X_{t}=X \cap H_{t}$. In [10], it is proved that an $(n-1)$-sphere $S$ in $E^{n}(n>5)$ such that each nonempty slice $S_{t}$ is either an $(n-2)$-sphere or a point has a 1-ULC complement whenever, for all $t, S_{t}$ is flat in both $H_{t}$ and $S$; subsequently, in [9] and [11] (see also [17]), $(n-1)$-spheres in $E^{n}(n>4)$ with 1-ULC complements were shown to be flat. The necessity of these conditions is discussed in [10] and [12]. Similarly, a 2-sphere in $E^{3}$ such that each nonempty slice is a point or a 1-sphere was earlier shown to be flat in [13] and [14] with each relying upon the 1-ULC taming theorem of [3]. In this note, we extend this work to the case $n=4$ by solving a similar question for a 4-cell; specifically, we prove the following:

THEOREM. $A$ 4-cell $B$ in $E^{4}$, such that each nonempty slice $B_{t}$ is either a point or a 3-cell, is flat whenever, for all $t, B_{t}$ is flat in $H_{t}$ and $\mathrm{Bd} B_{t}$ is flat in Bd $B$.

The proof relies upon a condition-first described to us by R. J. Daverman in 1976-under which an $n$-cell in $E^{n}$ is flat; Lemma 1 presents it. We include a proof because no reference contains the result; when $n>4$, it is superceded by the 1-ULC taming theorems of [3], [9], and [11]; yet when $n=4$, it has utility. (Daverman has pointed out that its hypotheses are strong enough to make the argument in Chernavskii [7] work too.)

LEMMA 1. Let $B$ be a 4-cell in $E^{4}$. If for each $\varepsilon>0$ there exists an $\varepsilon$-self-homeomorphism $h$ of $E^{4}$ supported in the $\varepsilon$-neighborhood of $E^{4}-B$ such that $h(\mathrm{Bd} B) \cap B=\varnothing$, then $B$ is flat. 
The proof of the theorem involves two other lemmas.

Lemma 2. Let $B$ be a 4-cell in $E^{4}$, and $T$ a 3-cell in $B$ with $\mathrm{Bd} T \subset \mathrm{Bd} B$ and Int $T \subset$ Int $B$ such that $B$ is locally flat at each point not in $\mathrm{Bd} T, \mathrm{Bd} T$ is flat in $\mathrm{Bd} B$, and $T$ flat in $E^{4}$. Then $B$ is flat.

Lemma 3. Let $P$ be a 4-cell in $E^{3} \times I$ such that $P_{0}$ and $P_{1}$ are points. Suppose $P$ is locally flat at each point of $\mathrm{Bd} P-\left(W \cup P_{0} \cup P_{1}\right)$ where $W$ is a countable union of 2-spheres in $\mathrm{Bd} P$ and suppose that for each 2-sphere $S$ in $W, S$ is contained in a horizontal hyperplane $H_{q}, S$ is flat in $H_{q}$, $S=\operatorname{Fr} P_{q}$, and $S$ is flat in $\mathrm{Bd} P$. Then $P$ is flat in $E^{4}$.

Lemma 2 may be regarded as giving sufficient conditions for the union of two 3-cells ( $T$ and a closed complementary domain of $\mathrm{Bd} T$ in $\mathrm{Bd} B$ ) in $E^{4}$ along their boundary to be flat, and so is related to [6] and [15] (see also [8]).

\section{Proofs of the lemmas.}

Proof of Lemma 1. Let $D=\mathrm{Bd} B, e: D \times I \rightarrow B$ be a collar on $D$ in $B$, and let $\left\{s_{i}\right\}$ be a decreasing sequence of numbers from Int $I$ which converges to 0 . Use the hypotheses to find a sequence of numbers $\varepsilon_{i}$ and a sequence of $\varepsilon_{i}$-self-homeomorphisms $h_{i}(i=1,2, \ldots)$ or $E^{4}$ such that $\varepsilon_{i}<\operatorname{dist}\left(e(D \times\{0\}), \quad e\left(D \times\left\{s_{i}\right\}\right)\right), \quad \varepsilon_{i+1}<\operatorname{dist}\left(D, h_{i}(D)\right), \quad h_{i}$ leaves $e\left(D \times\left\{s_{j}\right\}\right)$ fixed for all $j \leq i$, and $h_{i}(D) \cap B=\varnothing$. Then $\varepsilon_{i} \rightarrow 0$, $h_{i}(D) \cap h_{j}(D)=\varnothing$ for $i \neq j$, and $h_{i} \mid D$ converges uniformly to the identity. Let $q_{i} \in(0,1)$ be so close to 0 that $q_{i}<s_{i}$ and

$$
\operatorname{dist}\left\{h_{i+1} e(d, 0), h_{i+1} e\left(d, q_{i}\right)\right\}<\frac{1}{4} \operatorname{dist}\left\{h_{i+1}(D), h_{j}(D)\right\}
$$

for all $j \neq i+1$, and $d$ in $D$. Observe that the spheres $h_{i}(D)$ and $h_{i} e\left(D \times\left\{q_{i}\right\}\right)$ are all pairwise disjoint and "concentric".

Now use the product structure of $h_{i+1} e(D \times I)$ to find $\varepsilon_{i}$-self-homeomorphisms $F_{i}$ of $E^{4}$ such that

$$
F_{i} h_{i+1} e\left(d, s_{i}\right)=h_{i+1} e\left(d, q_{i}\right) \text { for all } d \text { in } D .
$$

and

$$
F_{i} h_{i} e\left(d, q_{i-1}\right)=h_{i} e\left(d, q_{i-1}\right) \text { for all } d \text { in } D .
$$

Then $F_{i} h_{i} e$ embeds $D \times\left[q_{i-1}, s_{i}\right]$ as the annulus between $h_{i} e\left(D \times\left\{q_{i-1}\right\}\right)$ and $h_{i+1} e\left(D \times\left\{q_{i}\right\}\right)$. 
Let $g_{i}: D \times[1 /(i+1), 1 / i] \rightarrow D \times\left[q_{i-1}, s_{i}\right]$ be a homeomorphism which preserves first coordinates and takes $D \times\{1 / i\}$ to $D \times\left\{q_{i-1}\right\}$. Now define $G: D \times I \rightarrow E^{4}-$ Int $B$ by

$$
G(d, 0)=d \text { for all } d \text { in } D
$$

and

(4) $G(d, t)=F_{i} h_{i} e g_{i}(d, t)$ when $1 /(i+1) \leq t \leq 1 / i$ and $d \in D$.

First observe that $G$ is continuous on $D \times(0,1]$ because each composition $F_{i} h_{i} e g_{i}$ is continuous on $\left.D \times[1 /(i+1), 1 / i)\right]$ and because (1) and (2) force these maps to agree whenever they have common domain; that is,

$$
F_{i+1} h_{i+1} e\left(d, q_{i}\right)=F_{i} h_{i+1} e\left(d, s_{i}\right)=F_{i} h_{i} e\left(d, s_{i}\right) \text {. }
$$

Next observe that $G$ is continuous on $D \times I$ because

$$
\operatorname{dist}\left(F_{i} h_{i} e g_{i}(d, q), e(d, 0)\right) \rightarrow 0 \quad \text { as } i \rightarrow \infty .
$$

Finally, $G$ is $1-1$ because the images $F_{i} h_{t} e g_{i}(D \times(1 /(i+1), 1 / i))$ are pairwise disjoint - they lie between different pairs of "concentric" spheres. $G$ is a collar on $B$, so $B$ is flat [2].

Proof of Lemma 2. Assume the hypotheses. Let $G$ be the decomposition of $\mathrm{Bd} B \times I$ into points and arcs of the form $\{x\} \times I$ with $x \in \mathrm{Bd} T$, let $\pi: \mathrm{Bd} B \times I \rightarrow \mathrm{Bd} B \times I / G$ be the decomposition map, and let $e$ : $\mathrm{Bd} B \times I / G \rightarrow B$ be a collar of $\mathrm{Bd} B$ in $B$ pinched at $\mathrm{Bd} T$ such that $\operatorname{diam} e \pi(\{x\} \times I) \leq \frac{1}{2} \varepsilon$ for all $x \in \mathrm{Bd} B$ and such that $e \pi(\mathrm{Bd} B \times I) \cap$ $T=\operatorname{Bd} T$. Let $K_{1}$ and $K_{2}$ denote the closed complementary domains of $\mathrm{Bd} T$ in $e \pi\left(\mathrm{Bd} B \times\left\{\frac{1}{2}\right\}\right)$. Since $B$ is a 4-cell and since $\mathrm{Bd} T$ is flatly embedded in $\mathrm{Bd} B, e \pi\left(\mathrm{Bd} B \times\left\{\frac{1}{2}\right\}\right)$ bounds a 4-cell with $\mathrm{Bd} T$ flatly embedded in its boundary; therefore there exists a homeomorphism $h$ of $E^{4}$ fixed on Bd $B$ such that $h\left(K_{1}\right)=K_{2}$. Set $T_{1}=h(T)$ and $T_{2}=h^{-1}(T)$; then $\operatorname{Bd} T_{i}=\operatorname{Bd} T$, Int $T \subset \operatorname{Int}(e \pi(\operatorname{Bd} B \times I))$, and each $T_{i}$ is flat. Also the union of $e \pi(\mathrm{Bd} B \times[0,1))$ and the compact set bounded by $T_{1} \cup T_{2}$ is $B$.

Now, according to [15], $T_{1} \cup T_{2}$ bounds a flat 4-cell $W$; hence there exists a $\frac{1}{2} \varepsilon$-self-homeomorphism $f$ of $E^{4}$ supported in the $\varepsilon$-neighborhood of $E^{4}-W$ such that $f(\operatorname{Bd} W) \cap W=\varnothing$, which means that $f$ is supported in the $\varepsilon$-neighborhood of $E^{4}-B$ and

$$
f(\mathrm{Bd} B) \subset\left(E^{4}-B\right) \cup(\mathrm{Bd} B-\mathrm{Bd} T) \cup \operatorname{Int}(e \pi(\mathrm{Bd} B \times I)) .
$$

Hence, using the pinched collar and the fact that $B$ is locally flat at points not in $\mathrm{Bd} T$, we can produce another $\frac{1}{2} \varepsilon$-self-homeomorphism $g$ of $E^{4}$ 
supported in $\operatorname{Int}(e \pi(\mathrm{Bd} B \times I)) \cup(\mathrm{Bd} B-\mathrm{Bd} T) \cup\left(E^{4}-B\right)$ such that $g f(\operatorname{Bd} B) \subset E^{4}-B$. Lemma 1 , with $h=g f$, now shows $B$ is flat.

Proof of Lemma 3. Assume the hypotheses. Let $W^{\prime}$ be the set of $t$ in $(0,1)$ such that $P$ is wild at some point of Bd $P_{t}$. Let $W^{*}$ be the closure of $W^{\prime}$ in $I$. Then $W^{*} \subset W^{\prime} \cup\{0,1\}$, so $W^{*}$ is closed and countable.

We want to show that $W^{*}$ equals the empty set; suppose it does not. Then by the Baire Category Theorem there exists an isolated point $q$ in $W^{*}$. In fact $q$ is in $W^{\prime}$. Now by using a pinched collar find a 4-cell $R \subset P$ such that $\mathrm{Bd} R \cap \mathrm{Bd} P$ is a neighborhood in $\mathrm{Bd} P$ of $\mathrm{Bd} P \cap H_{q}$, such that $R$ is locally flat modulo $\mathrm{Bd} P \cap H_{q}$, and such that $\mathrm{Bd} P \cap H_{q}=$ $\operatorname{Bd}\left(R_{q}\right)$. By hypotheses, $\mathrm{Bd} P \cap H_{q}$ is flat in $H_{q}$ and $\mathrm{Bd} P$; therefore it is flat in $\mathrm{Bd} R$ too. So according to Lemma $2, R$ is flat. Hence $P$ is locally flat at each point of $\mathrm{Bd} P-\left(W-\mathrm{Bd} P \cap H_{q}\right)$. It follows that $q$ is not in $W^{\prime}$, which is a contradiction. Therefore $W^{*}$ and $W^{\prime}$ are empty. Hence $P$ is locally flat at each point of $\mathrm{Bd} P-\left(P_{0} \cup P_{1}\right)$. It follows from [4] that $B$ is flat.

3. Proof of the theorem. Assume the hypotheses, and assume that $B \subset E^{3} \times I \subset E^{4}$ with $B_{0}$ and $B_{1}$ singleton sets. Let $J=[-1,1]$. We want to apply Lemma 1; so let $\varepsilon>0$ be given. Since $B_{t}$ is flat in $H_{t}$, there exists for each $t \in(0,1)$ a homeomorphism $h_{t}$ of $S^{2} \times E^{1}$ onto $H_{t}$ such that $h_{t} \mid S^{2} \times J$ is a bicollar on Bd $B_{t}$ with $h_{t}\left(S^{2} \times\{1\}\right) \subset H_{t}-B_{t}$. As in [10], there exists a countable set $D \subset I$ such that $s \in I-D$ implies the existence of monotone sequences $\{s(i)\}$ and $\{t(i)\}$ in $I$ converging to $t$ from above and below, respectively, such that $\left\{h_{s(i)}\right\}$ and $\left\{h_{t(i)}\right\}$ converge to $h_{t}$.

Fix $t$ in $I-D$, and let $p: E^{4} \rightarrow E^{3}$ denote projection. The local contractibility of the homeomorphism group of $E^{3}$ [5] at the point $p h_{t}$ shows that for each $\gamma>0$ there exist an integer $k$ and an isotopy $\left\{\phi_{q}\right\}$ of $E^{3}$ such that $\operatorname{dist}\left(\phi_{q}(x), p h_{t}(x)\right)<\gamma$ for all $q \in I$ and $x \in E^{3}, \phi_{1}=p h_{s(k)}$, and $\phi_{0}=p h_{t(k)}$. When $\gamma$ is small enough, an embedding $f_{t}:\left(S^{2} \times J\right) \times I$ $\rightarrow E^{4}$ may be defined by the rule

$$
f_{t}((a, b), c)=\left(\phi_{c}(a, b), c \cdot s(k)+(1-c) \cdot t(k)\right),
$$

possessing the following six properties:

$$
\begin{gathered}
f_{t}\left|\left(S^{2} \times J\right) \times\{1\}=h_{s(k)} ; \quad f_{t}\right|\left(S^{2} \times J\right) \times\{0\}=h_{t(k)} ; \\
f_{t}\left(\left(S^{2} \times\{1\}\right) \times I\right) \subset E^{4}-B ; \quad f_{t}\left(\left(S^{2} \times\{-1\}\right) \times I\right) \subset \text { Int } B ; \\
\operatorname{diam} f_{t}((\{s\} \times J) \times\{q\})<\frac{1}{4} \varepsilon \quad \text { for all } s \in S^{2}, q \in I ;
\end{gathered}
$$


and each set $f_{t}\left(\left(S^{2} \times J\right) \times\{q\}\right), q \in I$, is contained in a horizontal hyperplane.

Now let $Q=S^{2} \times J \times I$. There exists a countable collection $\left\{F_{i}\right\}$ of these embeddings (each $F_{i}$ equals some $f_{t}$ ) such that the union $\cup_{i=1}^{\infty} F_{i}(Q)$ $\cup \cup_{d \in D} H_{d}$ is a neighborhood of $\mathrm{Bd} B$ in $E^{3} \times I$. Let $K$ be the set of $q \in I$ for which $H_{q} \cap F_{i}(\operatorname{Int} Q)=\varnothing$ for all $i . K$ is countable because $D$ and $\left\{F_{i}\right\}$ are, and $K$ is closed because $\cup F_{i}($ Int $Q)$ is open.

Let $W$ be the union of the sets $(\operatorname{Bd} B)_{t}, t \in K$; then $W$ is a closed subset of $\mathrm{Bd} B$. Hence, as in the proof of Lemma 2 , one may use a pinched collar to find a map $e: \mathrm{Bd} B \times I \rightarrow B$ such that $e(x, 0)=x$ for $x \in \mathrm{Bd} B ; e(x, t)=x$ for $x \in W \cup B_{0} \cup B_{1}, t \in I ; \operatorname{diam}(e(\{x\} \times I))$ $<\frac{1}{2} \varepsilon$ for $x \in \mathrm{Bd} B ; \quad e \mid(\mathrm{Bd} B-W) \times I$ is an embedding; and when $t \in K, e(\mathrm{Bd} B \times I) \cap E_{t} \subset W$. Let $P$ be the 4-cell bounded by $e(\mathrm{Bd} B \times\{q\})$ where $q$ is so close to $D$ that $\mathrm{Bd} P$ is contained in the $\frac{1}{4} \varepsilon$-neighborhood of $\mathrm{Bd} B$. Also, assume without loss of generality that Bd $P \subset \operatorname{Bd} B \cup\left(\cup F_{i}(\right.$ Int $\left.Q)\right)$.

$P$ satisfies the hypotheses of Lemma 3 and is therefore flat in $E^{4}$. Hence there exists a $\frac{1}{2} \varepsilon$-self-homeomorphism $g$ of $E^{4}$, supported in the $\varepsilon$-neighborhood of $\mathrm{Bd} B$ such that $g(\mathrm{Bd} P) \cap P=\varnothing$. It follows that

$$
g(\operatorname{Bd} B) \subset\left(E^{4}-B\right) \cup\left(\cup F_{i}(\operatorname{Int} Q)\right) .
$$

So, because $g(\mathrm{Bd} B) \cap B$ is compact and contained in $\cup F_{i}($ Int $Q)$, there exists a finite subcollection $F_{1}, F_{2}, \ldots, F_{N}$, say, of the $F_{i}$ such that $g(\operatorname{Bd} B)$ $\cap B \subset \cup_{j=i+1}^{N} F_{i}($ Int $Q)$. We assume this subcollection is minimal; consequently, no point of $E^{4}$ lies in more than two of the sets $F_{i}(\operatorname{Int} Q)$, $i=1,2, \ldots, N$.

Now, for each $i=1,2, \ldots, N$, let $h_{i}$ be a $\frac{1}{4} \varepsilon$-self-homeomorphism of $E^{4}$ supported in $F_{i}(\operatorname{Int} Q)$, preserving fourth coordinates of $E^{4}$, and satisfying

$$
h_{i} h_{i-1} \cdots h_{i} g(\operatorname{Bd} B) \subset\left(E^{4}-B\right) \cup\left(\bigcup_{j=i+1}^{N} F_{i}(\operatorname{Int} Q)\right)
$$

Each $h_{i}$ is easily found as the composition of $F_{i}$ and a homeomorphism of $Q\left(=S^{2} \times J \times I\right)$ onto itself which leaves $\mathrm{Bd} Q$ fixed and only changes $J$ coordinates. Observe that $h_{N} \cdots h_{1} g(\mathrm{Bd} B) \cap B=\varnothing$.

Then because no point is moved by more than two of the $h_{i}$ 's, $h \equiv h_{N} \cdots h_{1} g$ is an $\varepsilon$-self-homeomorphism of $E^{4}$. Clearly $h$ is supported in the $\varepsilon$-neighborhood of $B$, so Lemma 1 shows $B$ is flat. 


\section{REFERENCES}

[1] F. Benson, A short proof of a Kirby flattening theorem, "Geometric Topology" Springer-Verlag, Berlin and New York, 1975.

[2] M. Brown, A proof of the generalized Schoenflies theorem, Bull. Amer. Math. Soc., 66 (1960), 74-76.

[3] R. H. Bing, A surface is tame if its complement is 1-ULC, Trans. Amer. Math. Soc., 101 (1961), 294-305.

[4] J. C. Cantrell, Almost locally flat embeddings of $S^{n-1}$ in $S^{n}$, Bull. Amer. Math. Soc., 69 (1963), 716-718.

[5] A. V. Chernavskii, Local contractibility of the homeomorphism group of a manifold, Soviet Math. Dok1., 9 (1968), 1171-1174.

[6] The k-stability of homeomorphisms and the union of cells, Soviet Math. Dokl., 9 (1968), 724-732.

[7] Locally homotopically unknotted imbeddings of manifolds, Soviet Math. Dok1., 9 (1968), 835-839.

[8] Homeomorphisms of euclidean space and topological imbeddings of polyhedra in euclidean spaces. III, Math. USSR-Sbornik, 4 (1968), 241-266.

[9] - The equivalence of local flatness and local 1-connectedness for imbeddings of $(n-1)$-dimensional manifolds in n-dimensional manifolds, Mat. Sb., 91 (133) (1973), 279-286-Math. USSR Sb., 20 (1973), 297-304.

[10] R. J. Daverman, Slicing theorems for $n$-spheres in Euclidean $(n+1)$-space, Trans. Amer. Math. Soc., 166 (1972), 479-482.

[11] Locally nice codimension one manifolds are locally flat, Bull. Amer. Math. Soc., 79 (1973), 410-413.

[12] R. J. Daverman and S. A. Pax, Cross sectionally simple spheres can be wild, General Topology, 10 (1979), 139-146.

[13] W. T. Eaton, Cross sectionally simple spheres, Bull. Amer. Math. Soc., 75 (1969), $375-378$.

[14] N. Hosay, A proof of the slicing theorem for 2-spheres, Bull. Amer. Math. Soc., 74 (1969), 370-374.

[15] R. C. Kirby, The union of flat $(n-1)$-balls is flat in $R^{n}$, Bull. Amer. Math. Soc., 74 (1968), 614-617.

[16] On the set of nonlocally flat points of a submanifold of codimension one, Ann. of Math., (2) 88 (1968), 281-290.

[17] T. M. Price and C. L. Seebeck III, Somewhere locally flat codimension one manifolds with 1-ULC complements are locally flat, Trans. Amer. Math. Soc., 193 (1974), $111-122$.

Received August 19, 1981 and in revised form January 14, 1983.

Pembroke State University

Pembroke, NC 28372 


\title{
PACIFIC JOURNAL OF MATHEMATICS EDITORS
}

\author{
Donald BabBitT (Managing Editor) \\ University of California \\ Los Angeles, CA 90024 \\ Hugo Rossi \\ University of Utah \\ Salt Lake City, UT 84112 \\ C. C. Moore and Arthur Ogus \\ University of California \\ Berkeley, CA 94720
}

\author{
J. DugundiI \\ Department of Mathematics \\ University of Southern California \\ Los Angeles, CA 90089-1113 \\ R. FINN and H. SAMELSON \\ Stanford University \\ Stanford, CA 94305
}

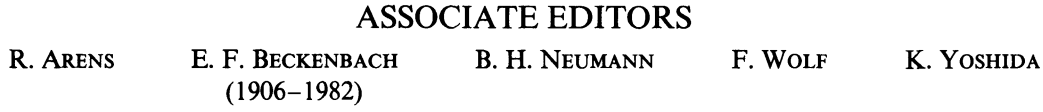

\section{SUPPORTING INSTITUTIONS}

UNIVERSITY OF ARIZONA
UNIVERSITY OF BRITISH COLUMBIA
CALIFORNIA INSTITUTE OF TECHNOLOGY
UNIVERSITY OF CALIFORNIA
MONTANA STATE UNIVERSITY
UNIVERSITY OF NEVADA, RENO
NEW MEXICO STATE UNIVERSITY
OREGON STATE UNIVERSITY

\author{
UNIVERSITY OF OREGON \\ UNIVERSITY OF SOUTHERN CALIFORNIA \\ STANFORD UNIVERSITY \\ UNIVERSITY OF HAWAII \\ UNIVERSITY OF TOKYO \\ UNIVERSITY OF UTAH \\ WASHINGTON STATE UNIVERSITY \\ UNIVERSITY OF WASHINGTON
}

The Supporting Institutions listed above contribute to the cost of publication of this Journal, but they are not owners or publishers and have no responsibility for its content or policies.

Mathematical papers intended for publication in the Pacific Journal of Mathematics should be in typed form or offset-reproduced (not dittoed), double spaced with large margins. Please do not use built up fractions in the text of the manuscript. However, you may use them in the displayed equations. Underline Greek letters in red, German in green, and script in blue. The first paragraph must be capable of being used separately as a synopsis of the entire paper. In particular it should contain no bibliographic references. Please propose a heading for the odd numbered pages of less than 35 characters. Manuscripts, in triplicate, may be sent to any one of the editors. Please classify according to the scheme of Math. Reviews, Index to Vol. 39. Supply name and address of author to whom proofs should be sent. All other communications should be addressed to the managing editor, or Elaine Barth, University of California, Los Angeles, California 90024.

There are page-charges associated with articles appearing in the Pacific Journal of Mathematics. These charges are expected to be paid by the author's University, Government Agency or Company. If the author or authors do not have access to such Institutional support these charges are waived. Single authors will receive 50 free reprints; joint authors will receive a total of 100 free reprints. Additional copies may be obtained at cost in multiples of 50 .

The Pacific Journal of Mathematics is issued monthly as of January 1966. Regular subscription rate: $\$ 132.00$ a year (6 Vol., 12 issues). Special rate: $\$ 66.00$ a year to individual members of supporting institutions.

Subscriptions, orders for numbers issued in the last three calendar years, and changes of address should be sent to Pacific Journal of Mathematics, P.O. Box 969, Carmel Valley, CA 93924, U.S.A. Old back numbers obtainable from Kraus Periodicals Co., Route 100, Millwood, NY 10546.

The Pacific Journal of Mathematics ISSN 0030-8730 is published monthly by the Pacific Journal of Mathematics at P.O. Box 969, Carmel Valley, CA 93924. Application to mail at Second-class postage rates is pending at Carmel Valley, California, and additional mailing offices. Postmaster: Send address changes to Pacific Journal of Mathematics, P. O. Box 969, Carmel Valley, CA 93924.

PUBLISHED BY PACIFIC JOURNAL OF MATHEMATICS, A NON-PROFIT CORPORATION

Copyright $(1983$ by Pacific Journal of Mathematics 


\section{Pacific Journal of Mathematics}

Vol. 108, No. $2 \quad$ April, 1983

Enrique Atencia and Francisco Javier Martin-Reyes, The maximal ergodic Hilbert transform with weights $\ldots \ldots \ldots \ldots \ldots \ldots \ldots \ldots \ldots . \ldots 257$

Bruce Blackadar, The regular representation of local affine motion

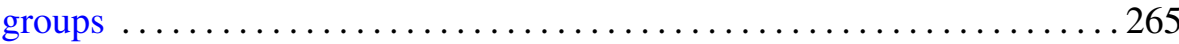

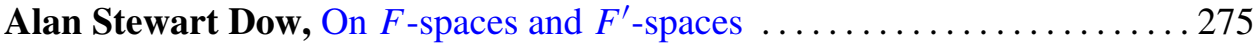

Yoshifumi Kato, On the vector fields on an algebraic homogeneous space . . 285

Dmitry Khavinson, Factorization theorems for different classes of analytic functions in multiply connected domains $\ldots \ldots \ldots \ldots \ldots \ldots \ldots \ldots 295$

Wei-Eihn Kuan, A note on primary powers of a prime ideal . . . . . . . . 319

Benjamin Michael Mann and Edward Yarnell Miller, Characteristic classes for spherical fibrations with fibre-preserving free group

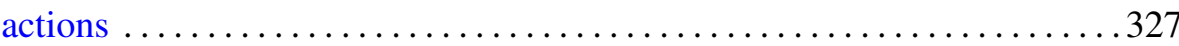

Steven Alan Pax, Appropriate cross-sectionally simple four-cells are flat . . . 379 R. K. Rai, On orthogonal completion of reduced rings ................ 385

V. Sree Hari Rao, On random solutions of Volterra-Fredholm integral

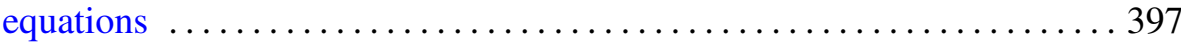

Takeyoshi Satō, Integral comparison theorems for relative Hardy spaces of solutions of the equations $\Delta u=P u$ on a Riemann surface $\ldots \ldots \ldots . .407$

Paul Sydney Selick, A reformulation of the Arf invariant one $\bmod p$ problem and applications to atomic spaces

Roelof Jacobus Stroeker, Reduction of elliptic curves over imaginary quadratic number fields

Jacob Towber, Natural transformations of tensor-products of representation-functors. I. Combinatorial preliminaries

James Chin-Sze Wong and Abdolhamid Riazi, Characterisations of amenable locally compact semigroups 